\title{
CORONA MODELLING FOR THE CALCULATION OF TRANSIENTS ON TRANSMISSION LINES
}

\author{
Adam Semlyen \\ University of Toronto \\ Toronto , Ontario, Canada
}

\author{
Huang Wei-Gang \\ Tsinghua University \\ Beijing, China
}

\begin{abstract}
The paper presents a mathematical corona model and its use for transmission line modelling with corona and, if desired, with frequency dependence of its parameters. The corona model has been developed from macroscopical physical laws, reflecting relations between charge, electric field intensity and voltage. Space charges, their development and their displacement are taken into account. The parameters of the model are determined so that the simulated $q-v$ curves closely fit those available from measurements. The corona model is then used as an element of the discretized transmission line model. This consists either of longitudinal inductances or of travel delay modules. Modelling of frequency dependence is optional. The line equations are integrated using the trapezoidal rule, so that Norton equivalents are obtained for the two line ends for the purpose of interfacing with the external system. Thus the model is fully compatible with an Electro-Magnetic Transients Program (EMTP).
\end{abstract}

\section{INTRODUCTION}

The calculation of Electro-Magnetic Transients (EMT) in transmission systems was initially performed with lossless transmission line models [1]. A more realistic transmission line model has frequency dependent (but still linear) longitudinal parameters and voltage dependent nonlinear transversal parameters due to corona. Both are important in many practical problems. However, the calculation of EMTs is greatly simplified if linearity is assumed and therefore the problem of simulation with frequency dependent parameters was first solved [2],[3],[4]. This work, essentially performed in the nineteen-seventies, can now be considered complete, at least for overhead lines. Corona modelling has however progressed much more slowly. In 1975 Gela et al. have reported on a rather sophisticated procedure [5] in which corona was modelled by algebraically defined branches of measured $q-v$ curves. The more recent reference [6] reports on a similar approach. We consider the work described in references [5] and [6] as fairly complete but the predefined corona model too rigid. We would rather see it representing physical phenomena as they evolve, and this is the main characteristic of the model we are presenting in this paper.

The physical phenomenon of corona is very complex. It includes ionization, effects of mobility (of electrons, of positive and negative ions), diffusion, deionization (recombination, attachment, etc.) and the mutual effect of space charges and electric field, too complicated to be fully described by mathematical equations. No model has yet evolved which permits the representation of the complex phenomena in a com-

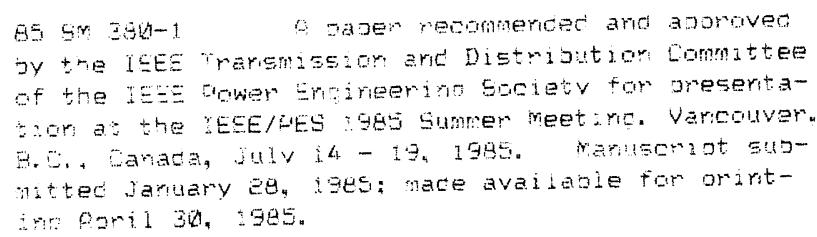

puter program for EMT simulation. Clearly, simplifications are necessary. A simplistic approach is the one of Ref.[7], where corona is represented by voltage dependent nonlinear capacitances, and lumped resistances are used to reproduce losses. It is certainly questionable to what extent results obtained by Peek for sinusoidal voltages could be used for transients. Also, losses on a resistance as used in [7] depend on voltage only, while corona losses are proportional to frequency since they are given by the area of the $q-v$ curve. The models of Ref.[8] and Ref.[9] are more elaborate and realistic. They use surface charges for corona modelling. In the present paper we model corona by both space and surface charges, which is closer to reality.

Basic to the corona model developed in this paper is the concept of state. It is determined by the space charges around the conductor. They may form a number of positive or negative layers, completely defined by their radii, which thus represent state variables. Their number is small. All other variables can be derived from these state variables, or an alternate set of state variables (e.g. space charges) can be defined. By storing a few state variables, at each time step the complete physical condition of the local corona is available for updating.

The macroscopic model used in this study is not intended to reproduce the details of the microscopic phenomena. For instance, ions and electrons are not considered separately and the movement of these particles is also ignored as long as the resultant state is stationary. Instead, space charges are considered, always represented by the same algebraic variable which can be positive or negative. Non-uniformities of the corona layer are ignored for the purpose of fully symmetrical representation and the movement of charges is described by the simple assumption of discontinuous friction-like behaviour, corresponding to the idealized assumption of a sudden increase of mobility from zero to infinity above a critical electric field intensity. Thus the model is not a true representation of the physical phenomena, only a substitute which produces external $q-v$ characteristics similar to those available from measurements [10]. But, since measurements are available only for a few conductor configurations, it is expected that the macroscopic model will generate correct $q-v$ traces for more general conditions. In fact, $q-v$ curves are available for only a few types of inputs, and in the case of an arbitrary transient we may have $q-v$ traces not yet produced by tests. Therefore, the macroscopic corona model appears to be indispensable for the simulation of other than the simplest transients.

The corona model contains a number of numerical parameters. These have to be chosen so that physical experiments [10] could be closely reproduced with the model. Such experiments indicate that at fast impulses the measured $q-v$ curves may significantly differ from those for slower impulses. Therefore, a dynamic corona model has also been developed.

The corona model serves as part of a discretized transmission line model which itself becomes part of a system. Two alternatives have been used for modelling the longitudinal ele ments of the line. In one these are represented by an inductance, in the other by a lossless delay segment. For frequency dependent impedance modelling $R, L$ elements are added, as described in Ref.[11]. For compatibility with an ElectroMagnetic Transients Program (EMTP), the resultant line model 
yields Norton equivalents which permit interfacing with external components. Three phase lines can be readily represented with direct phase domain modelling.

\section{THE CORONA BRANCH}

The Static Model

\section{Basic Assumptions}

It is assumed that the corona geometry is cylindrical. This assumption is properly justified in Ref.[9]. The phenomena of ionization do not develop beyond a fictitious cylinder of radius $R$ (see Fig. 1). Thus $R$ can be selected arbitrarily, large enough to contain the ionized region but sufficiently small for nearly cylindrical symmetry of the inside electric field. At $R$ we consider the reference for voltages, i.e. $v=0$ and all calculations are performed starting from $x=R$. We will calculate for all $x$ the electric field intensity $E$ and its integral, the voltage $v$, moving backward to the conductor of radius $r$. First there is a nonionized region. Then, starting from radius $X$ there may be an ionized region consisting of one or several corona layers of space charges of alternating polarities. At $X$ there is a shell of surface charges $Q$, representing a concentration of space charges in the real corona. When corona advances, i.e. $X$ moves outward, then at $x=X, E$ has the critical value

$$
E_{C R}=A+B / X
$$

where $A$ and $B$ are as yet undetermined constants. They are related to each other by the condition

$$
E_{C R_{\bullet}}=A+B / r
$$

where $E_{C R_{0}}$ is the critical field intensity at the conductor surface, $30 \mathrm{kV} / \mathrm{cm}$. Thus only one free parameter remains for identification.

The calculations assume that the charge $q$ is given. Its negative is on the cylinder of radius $R$. If $q$ increases, so will $E$, and $X$ moves forward. Behind $X$ there will be space charges of density $\rho$, moving out from the conductor. The electric field intensity in the ionized region is assumed not to exceed a critical value

$$
E_{c r}=a+\frac{b}{x}
$$

which is much smaller than $E_{C R}$.

The space charges will not move, assuming that the transients examined are not very slow, unless $E$ in any direction exceeds $E_{c r}$. This assumption holds for both $\rho$ and $Q_{s}$. In consequence, if the polarity of $E$ is reversed, $E$ will have to exceed $E_{c r}$ in the opposite direction and then a new layer of corona will advance, gradually replacing the previous one. If it does not advance sufficiently then we will have two layers, and so on. For the inception of a new corona layer a sufficiently strong field is

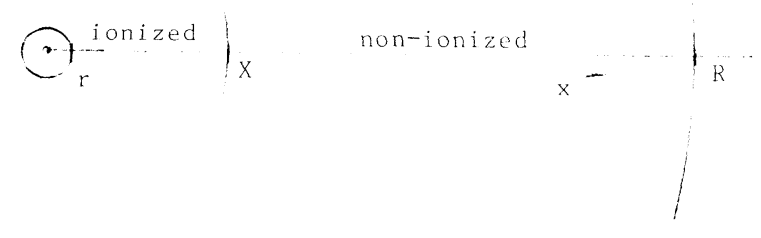

Fig. 1 Radial variables. needed on the conductor surface. We can always assume that such a field is available because only a negligibly small voltage is needed for such a field over a very small distance. Therefore we will not care any more about this field and will ignore it in our calculations.

The assumption that an $E_{c r}$ exists corresponds to the movement of space charges under the effect of an electric field as discussed in the introduction. In general, the charges would move with any field magnitude in the ionized region. However, if the reaction force is constant (rather than a general function of velocity), then this friction like reaction will permit the complete displacement of charges, without any delay, once the applied field attains the value of $E_{c r}$.

By having assumed critical field magnitudes $E_{C R}$ and $E_{c r}$, any slow charge displacement has been eliminated from the model. Therefore, the results of simulation will not apply for slower phenomena, such as dynamic overvoltages, where the drifting of charges is important. For such cases the model has to be further developed.

The assumption in (1) that $E_{C R}$ is a function of $X$ is based on the observation that a constant $E_{C R}$ yields a breakdown voltage which is much higher than the measured values. Since $E_{c r}$ $<<E_{C R}$ we will assume for simplicity of our argument that $E_{c r}$ $=0$. Then, with $E_{C R}=$ const, the breakdown occurs at the critical radius $R / e(e=2.718)$ at the voltage $E_{C R} R / e$. Since this is too large, $E_{C R}$ must be a decreasing function of $X$ which also reduces the breakdown voltage, as shown in Appendix 1 . The particular forms of equations (1) and (3) are chosen for computational convenience. The parameters $A$ and $B$ are of course functions of polarity (among other things, such as humidity, rain, pressure) and $a$ and $b$ may also depend on polarity. For simplicity of presentation we will however omit related notational details.

As mentioned, the calculations are performed starting with a given $q$ and proceed from outside in, to give $v=f(q)$. Experiments yield however $q-v$ curves with voltage as input. For the simulation of experiments the relation $v=f(q)$ has therefore to be inverted numerically. This is best done using the secant method. However for corona modelling as part of a transmission line model, the direct relation $v=f(q)$ is needed, as it will become apparent later.

\section{Mathematical Corona Representation}

The First Corona Layer. Fig. 2 shows the critical field intensities as well as the actual value $E$. In the non-ionized region it is

$$
E=\frac{q}{2 \pi \epsilon x}
$$

The intersection of the two characteristics, corresponding to equations (1) and (4) determine the boundary $X$ of the ionized layer. At the boundary the surface charge is

$$
Q_{s}=2 \pi \epsilon X\left(E_{C R}-E_{c r}\right)
$$

It is proportional to the difference $E_{C R}-E_{c r}$ and is visualized in Fig. 2 by the discontinuity in $E$ at $x=X$.

In the ionized region the space charge density $\rho$ is given by

$$
p=\epsilon \operatorname{div} E
$$

or, in cylindrical coordinates,

$$
\rho=\epsilon \frac{d(x E)}{x d x}
$$

With $E=E_{c r}$ of (3), equation (6) yields 


$$
\rho=\frac{\epsilon a}{x}
$$

The voltage of the conductor is obtained by integrating $E$ from $r$ to $R$.

The Second Corona Layer. If $q$ decreases then $E$ decreases proportionally in the non-ionized region, as shown by the dashed line for $E^{\prime}$ in Fig. 2. All space and surface charges remain trapped. The discontinuity at $X$ remains of the same magnitude, but is shifted downward. The line $E^{\prime}$ may now intersect the lower characteristic of $E_{c r}$ at a point $x_{2}$. This indicates that a second corona layer, of opposite sign, has started and progresses in the space of the former positive layer. Its progress has to be monitored, step by step, and when $x_{2}$ reaches $x_{1}=X$, the surface charges $Q_{s}$ decrease and are eventually replaced by surface charges of opposite polarity so that a negative $E$ builds up until it reaches the corresponding negative critical value. Then the negative front can advance into the non-ionized region.

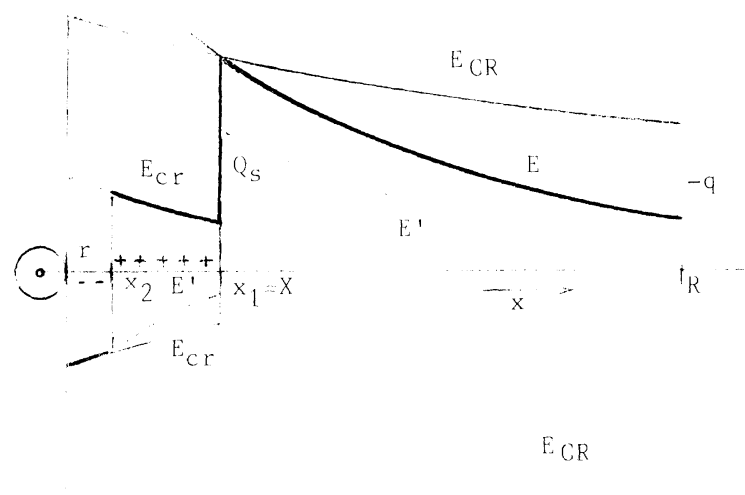

Fig. 2 Critical and actual values of $E$.

Fig. 3 shows the $q-v$ curve corresponding to the phenomena described above. Since the model is static, it does not matter how the sequence of input quantities relates to time. Thus, at the lower part of the figure, the plots are arbitrarily chosen as piecewise linear. The sequence of points OABCD'E' corresponds to curves (a), and OABCDEFGH to curves (b). Curve (a) represents an input with decreasing swing amplitudes, so that the negative corona does not completely replace the positive one. Curve (b) shows an input with increasing swing amplitudes so that the negative corona layer fully replaces the former positive layer and then continues to advance as negative corona into the non-ionized region.

In the next section we will consider the multi-layer case. Then the two layers we have considered now will have in front of them other layers (see Fig.4). Therefore, when the last layer fully replaces the one over which it advances, it will simply merge with a layer of the same polarity existing ahead of it. Thus the number of layers is reduced by two: this process of cancellation eliminates layers in pairs.

The General Case. For computer implementation of the corona model the multi-layer situation of Fig. 4 is considered. There are $k$ layers of alternating polarity. Only the pair closest to the conductor is active, while $k-2$ layers have fixed charges. Eventually, if layer $k$ cancels layer $k-1$ and merges with layer $k-2$, the latter, together with layer $k-3$, become the active pair. At the beginning we have particular cases with only one or two layers.

The computations start with a given charge $q$ the negative of which is on the boundary cylinder of radius $R$ where we assume $v=0$. Since $Q$, and all $k-1$ space charges are fixed, they are known, by equations (5) and (7). Therefore, starting with $E$
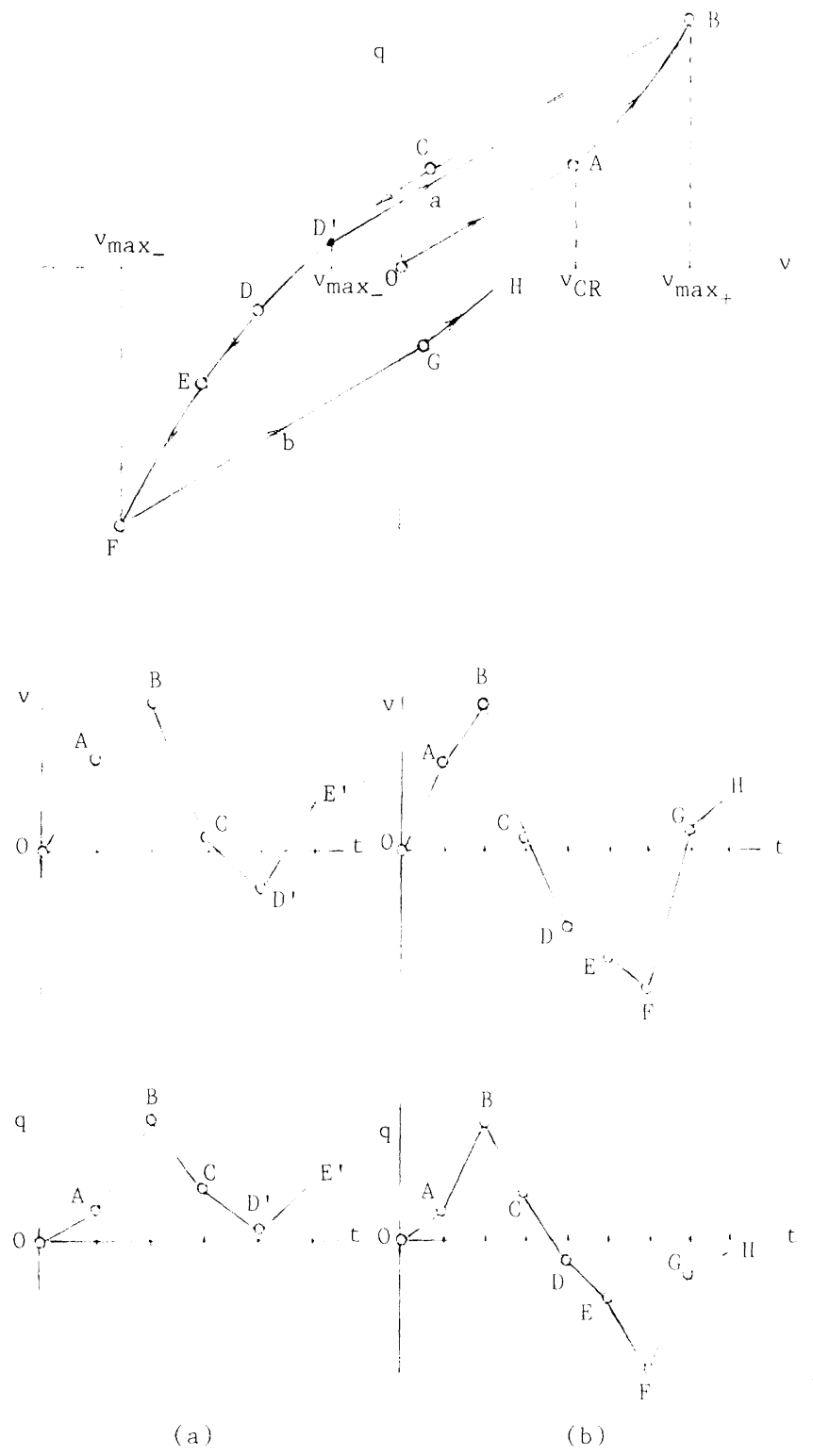

Legend:

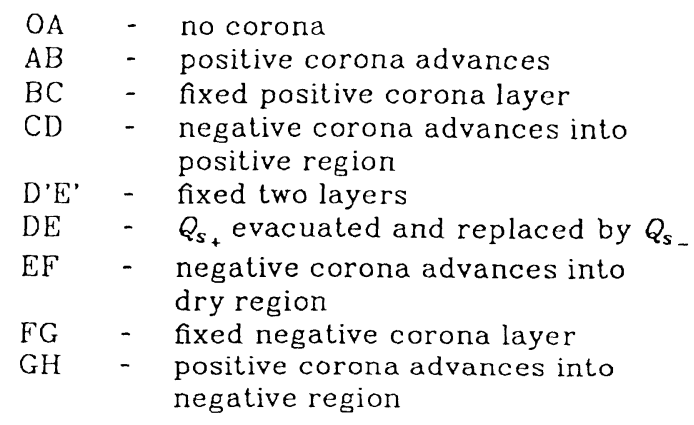

Fig. $3 \quad q-v$ curve for two-layer corona

(a) Second layer does not fully replace first layer.

(b) Second layer fully replaces first layer.

given by (4), we can obtain $E$ at $x_{k-1}$ using (6) and, by integration, the voltage at that point. As a matter of practical significance, it saves time using superposition for this calculation and separating the effects of the total charge $q$ and of the fixed space charges, since then only the former has to be recalculated 


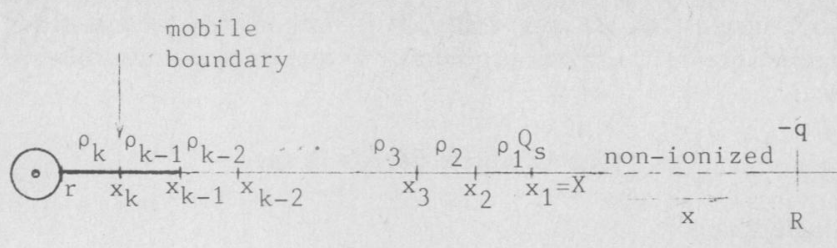

active passive layers
layer
pair

Fig. 4 Multilayer corona.

at each time step using equation (4) for $x$ near the conductor. Integration of (6) from $x_{j}$ using (7), yields

$$
E=a+\left(E_{j}-a\right) \frac{x_{j}}{x}
$$

This gives us $E_{j+1}$ for recursive calculation of $E$ and, for $j=k-1$, the profile of $E$ needed for determining the boundary $x_{k}$ of the newest layer. We note that $E$ in both (3) and (8) has the same "hyperbolic" form, so that the intersection point of the two curves is obtained by a simple formula.

We conclude this section with the remark that, if the new value of $x_{k}$ results smaller than the previous one, then it is left unchanged; the profile of $E$ will then move toward the other limit $E_{c r}$, and if this is reached (which would always happen first at the conductor surface) then a new layer would start so that $k$ has to be incremented by 1 .

With $E$ known by expressions of "hyperbolic" form, it is easy to obtain closed form logarithmic expressions for $v$ on the conductor surface.

\section{The Dynamic Model}

Experimental results indicate that the $q-v$ characteristic for fast impulses [10] or high frequency a.c. [12] is narrower than for slow ones. This is shown in detail in Fig.5. Curve $a$ corresponds to any impulse with a rise time slower than approximately $10 \mu \mathrm{s}$. This is the static case. Curve $b$ gives the $q-v$ characteristic for a faster impulse. It is narrower than curve $a$, and a faster impulse would have produced an even narrower result. This is the dynamic case. It can be seen from the figure that it has an increased critical voltage. In the mathematical model this can be achieved by multiplying $E_{C R}$ by the factor

$$
1+K|\dot{q}|
$$

which increases $E_{C R}$ as $\dot{q}$ the rate of change of the corona input $q$ becomes significant.

In conclusion, the dynamic corona model gives $v$ as a function of $q$ and $\dot{q}$ :

$$
v=f(q, \dot{q})
$$

\section{Simulation of $q-v$ Curves}

The corona model has three parameters for each polarity, $A, a$ and $b$ (since $B$ is related to $A$ by equation (2)), which can be used to make the model conform to experimental data. We would like to refer, in particular to the set of measurements reported in Ref.[10] containing $q-v$ curves for a wide range of conditions.

The parameters can be identified by a trial-and-error procedure or any other method. Fig. 6 shows the result of the parameter identification. It represents both a measured $q-v$ curve and the simulated one for an impulse. For $1.823^{\prime \prime}$ conductor in $5.5 \mathrm{~m} \times 5.5 \mathrm{~m}$ mesh cage, $2.5 / 60 \mu \mathrm{s}$ positive impulse, the

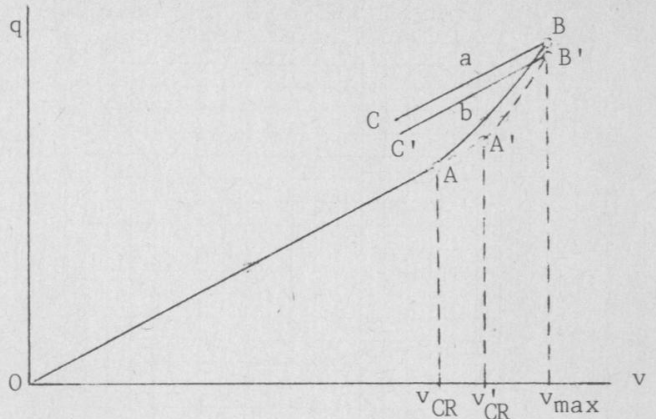

Fig. $5 q-v$ curves for impulse voltages

(a) Slow impulse, static model.

(b) Fast impulse, dynamic model.

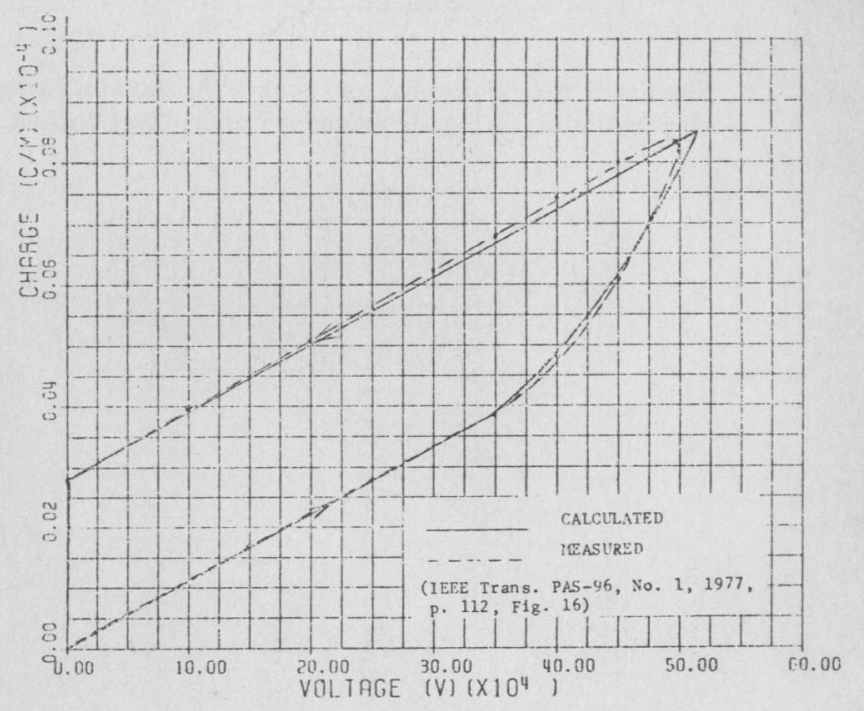

Fig. 6 Measured and simulated $q-v$ curve.

parameters of the model are: $r_{o}=0.023 \mathrm{~m}, R=3.5 \mathrm{~m}, A=150$ $\mathrm{kV} / \mathrm{m}, B=66 \mathrm{kV}, a=80 \mathrm{kV} / \mathrm{m}$ and $b=60.7 \mathrm{kV}$.

After parameter identification the model is complete and can be used for simulation purposes. Fig. 6 has served for the validation of the static model. Figures 7 and 8 represent simulated $q-v$ curves for oscillatory inputs as in Fig. 3. We are not aware of any measurements with this type of input and would hope that such measurements will become available and will further validate the corona model. It is interesting to note that the model displays hysteresis-like features, as expected because of the assumed charge movement which appears like governed by discontinuous friction. Minor loops are possible and the losses result from the integral

$$
w=\int v i d t=\int v d q
$$

Fig. 9 shows a simulation of a fast impulse $(0.5 / 60 \mu \mathrm{s}) q-v$ curve, using the dynamic corona model. The apparent (dynamic) critical voltage is $386 \mathrm{kV}$, while the static one is 350 $\mathrm{kV}$ for this case. One can choose proper parameters to fit measured curves if they become available.

We note that at the return slope of the $q-v$ curve the corona model assumes that the charges do not move. Therefore, a straight line corresponds to this portion on figures 3 and 5 . In reality, if the corona impulse is slow or very slow, the charges eventually disperse producing a curving of the return slope. The modelling of this phenomenon has not been attempted in this 


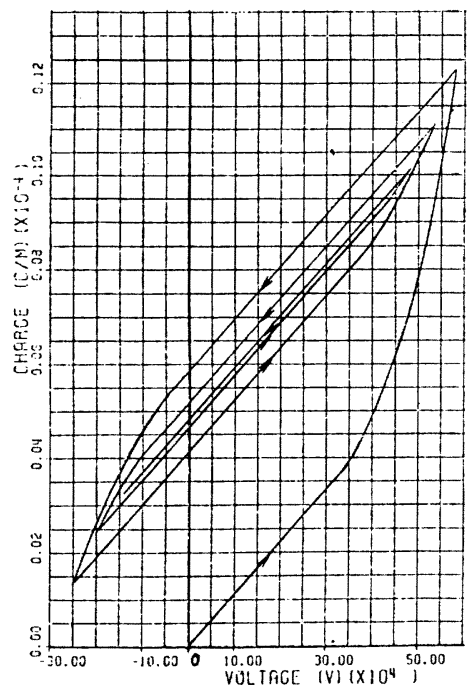

Fig. 7 Simulated $q-v$ curve for an input of decreasing swing amplitudes, as in Fig. 3a producing multi-layer corona.

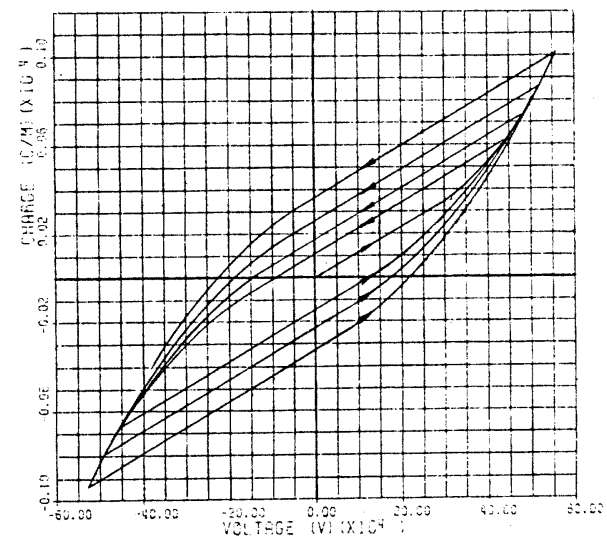

Fig. 8 Simulated $q-v$ curve for an input of increasing swing amplitude, as in Fig. 3b, producing corona of alternating polarity.

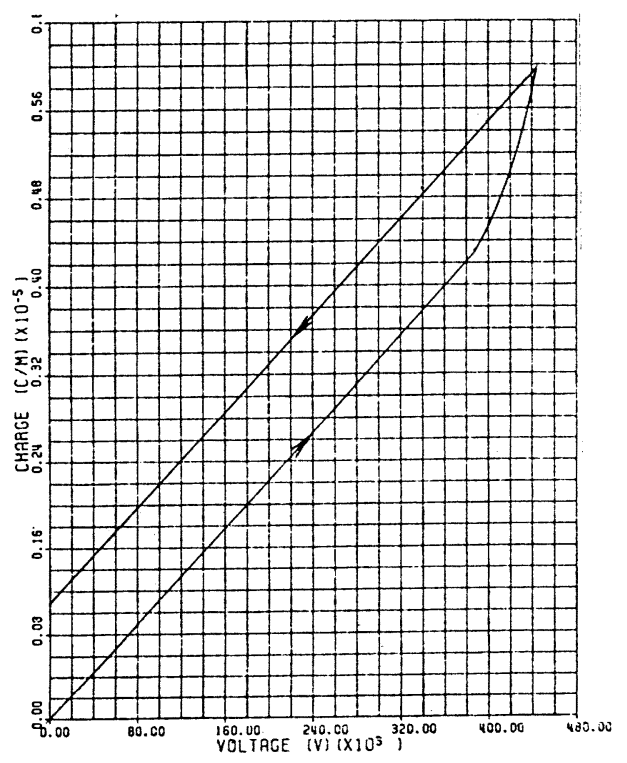

Fig. 9 Dynamic $q-v$ curve simulation. work because of its complexity. It is probably of second order significance in the usual problems of transients on transmission lines.

\section{The Complete Corona Branch}

\section{Single Phase}

Our corona model has so far been confined in a cylindrical space of radius $R$. This has to be completed by the external capacitance $C_{R}$, as shown in Fig.10. Since both at the inner and at the outer surface of the cylinder the air is not ionized, the exact value of $R$ is of no significance, as already mentioned, but must of course be kept small so that the cylinder approximates well an equipotential surface.

If the voltage difference inside the cylinder is $f(q, \dot{q})$ and outside the cylinder $q / C_{R}$, then the conductor voltage is given by

$$
v=F(q, \dot{q})=f(q, \dot{q})+\frac{q}{C_{R}}
$$

This model is used in the lumped parameter model of a transmission line with corona, to be described later. It represents the transversal branches of the model, while the longitudinal branches are pure inductances in the lossless case and are supplemented by $R, L$ branches if losses and frequency dependence are represented. In the travelling wave line representation, in the lossless case the ideal delay segment is equivalent to the series inductance and the ideal line capacitance $C_{r}$ in parallel. This is made available by subtracting it from the complete corona model for lumped parameter lines, which means adding $-C_{r}$ in parallel, as shown in Fig.10. Therefore, we need two parallel branches, $-C_{r}$ and the one corresponding to equation (12), for the complete modelling of the transversal element in a travelling wave transmission line representation. Of course, if there is no corona, this transversal element will automatically vanish.

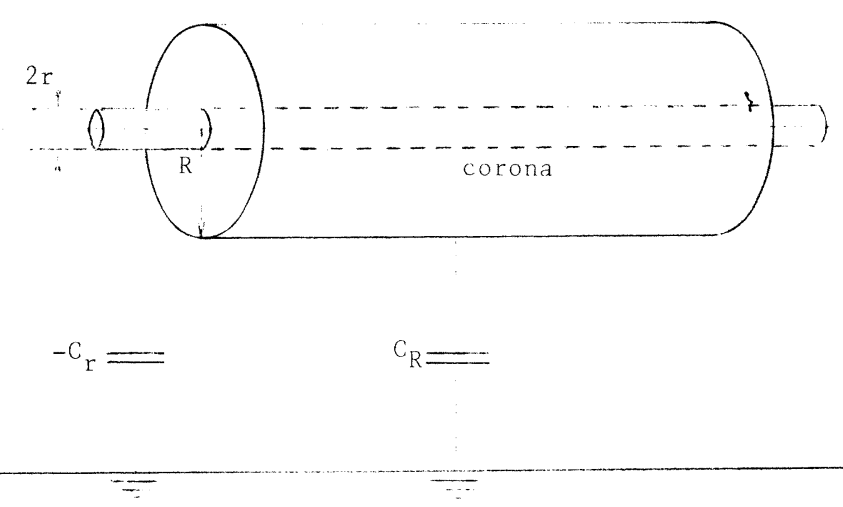

Fig. 10 Complete transversal branch of single phase conductor.

\section{Three Phase}

The modelling problems discussed above remain valid in the three phase case, but require the use of matrix equations to reflect the more complex conditions shown in Fig. 11. In particular, equation (12) has to be interpreted as representing diagonal entries for the corona models $f(q, \dot{q})$, while the term representing the voltage vector across the external capacitances should be interpreted as the product $C_{R}^{-1} \times q$, where $C_{R}$ is now the capacitance matrix and $q$ the vector of charges. 


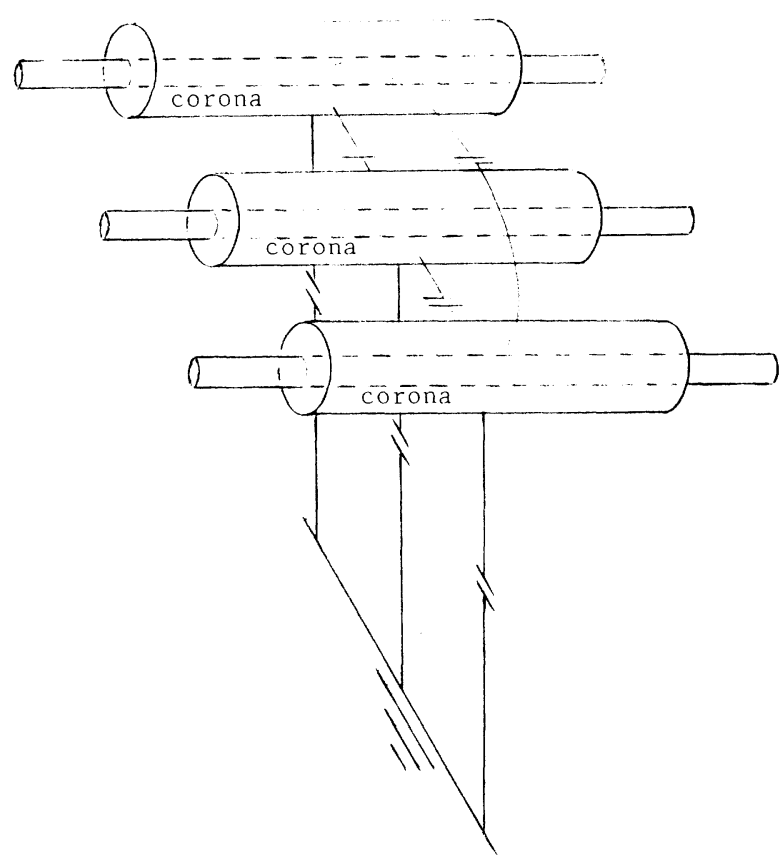

Fig. 11 Three phase corona modelling.

\section{TRANSMISSION LINE MODELLING WITH CORONA}

\section{Requirements and Alternatives}

The basic requirement for the transmission line model with corona is EMTP compatibility, i.e. it should have the interfacing capability with an Electro-Magnetic Transients Program using Norton equivalents for line and lumped component modelling [13]. Therefore care is taken for the line model to appear at both ends as a Norton equivalent.

A second requirement is related to the nonlinear nature of the corona branch. Norton equivalents require implicit integration, preferably using the trapezoidal rule. This however leads to local iterations at each corona branch. It is important that this process converges rapidly. At the same time the decoupling effectively afforded by the physical time delay between line ends should be preserved as much as possible even if the line is represented by lumped parameters.

The longitudinal branches of the transmission line can be represented by either lumped $R, L$ elements (a pure inductance in the lossless case) or by ideal delay segments supplemented by $R, L$ elements if frequency dependence is modelled. The number of segments needed for discretization depends on the type of transient being simulated.The steepest slope must be subdivided into a sufficiently large number of segments, not only for accuracy but also because the $L, C$ loops of a too coarse model create eigenvalues which will be excited by the high frequency input if the natural frequency is not sufficiently high.

There are two options for line modelling: lossless and frequency dependent. Especially for slower (switching) transients, frequency dependent modelling is important. In addition, frequency dependent modelling provides the necessary damping at high frequencies to remove the parasitic oscillations mentioned above. Thus it permits to reduce the number of line segments and thereby the cost of frequency dependent modelling.

Finally, the modelling can be three phase or single phase for preliminary studies. In many practical situations, single phase studies will be sufficiently revealing for assessing the effect of corona on transients on lines and equipment.

\section{The Lumped Parameter Model}

Fig. 12 illustrates the lumped parameter approach for the modelling of a single phase transmission line with corona. The line is subdivided into segments with the currents $i$ and the voltages $v$ or the charges $q$ as state variables; see Fig. 12a. In Fig. $12 \mathrm{~b}$ the state variables are all denoted by $x$ with an odd integer subscript for $v$ or $q$ and an even integer subscript for $i$. For any state variable $x_{k}$ a state equation of the form

$$
\dot{x}_{k}=f\left(x_{k-1}\right)-f\left(x_{k+1}\right)
$$

can be written for the lossless line with static corona modelling, indicating that $\dot{x}_{k}$ is directly related to the two neighbouring state variables. Concretely, equation (13) becomes

$$
\dot{q}_{k}=i_{k-1}-i_{k+1}
$$

or

$$
i_{k}=f\left(q_{k-1}\right)-f\left(q_{k+1}\right)
$$

These relations are shown by the graph of Fig. $12 \mathrm{c}$ which is part of Fig. 12b. If the functions representing the corona branches were linear, then all equations (13) could be discretized by the trapezoidal rule and assembled into a set of linear equations with a tridiagonal coefficient matrix. The solution of these equations at each time step would be cumbersome. In the nonlinear case the solution of the problem would be computationally complicated and expensive. A simple and efficient solution method consists in solving equations (13) by a "sweeping" procedure, described in the following.

Trapezoidal discretization of (13) yields

$$
x_{k}=f\left(x_{k-1}\right)-f\left(x_{k+1}\right)+\text { history }
$$

The graphs of Fig. 12b and c are applicable for equation (14) as well. Now, when we update $x_{k}$ by sweeping from left, then $x_{k-1}$ is the value just calculated, but $x_{k+1}$ is the the most recent old value; a similar statement applies when sweeping from right. The sweeping is made alternatively in both directions, within
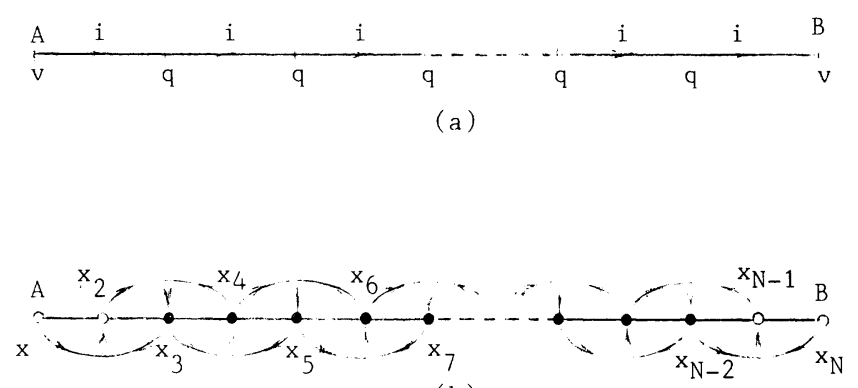

(b)

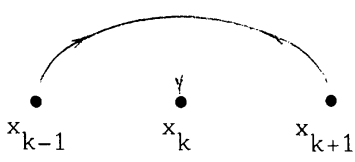

(c)

Fig. 12 Lumped parameter approach for line modelling.

(a) The segmented line with state variables $i, v$ and $q$.

(b) Scheme for writing state equations.

(c) Graph for the equation for state variable $x_{k}$. 
each time step $\Delta t$ and corresponds to a successive replacement or Gauss-Seidel type algorithm. Its convergence is linear but very fast, fully consistent with the theoretical analysis of Appendix 2. Numerical testing has confirmed that the error decreases faster for smaller time steps, typically by one order of magnitude in each simple sweep.

When the sweeping reaches either end $A$ or $B$ of the line, $x_{1}$ and $x_{N}$ are given as voltages and a Norton equivalent is obtained from the end equation. The variables are $v$ and $i$ and are shown in Fig. 12b as empty circles to indicate that these are terminal variables. by combining these line Norton equivalents with those of the external components, we obtain $v$ and $i$ which are thus known for the sweep starting in the other direction.

While the lumped parameter approach with sweeping will give a fast solution of all nonlinear corona equations together with the longitudinal line equations and the external components, the problem becomes too complicated if the line is modelled with frequency dependent parameters. The travelling wave approach, to be presented next, appears to be more appropriate for frequency dependent line modelling and assures a de facto time decoupling between all transversal branches. Local iterations, because of the nonlinearity of the corona model, can however not be avoided.

\section{The Travelling Wave Approach}

If travelling waves are used in the longitudinal branches a time delay $\tau_{0}$ will model each segment, with the $R, L$ model for frequency dependent "shaping" in series, as shown in Fig. 13. The wave shaping circuit consists of parallel $R, L$ branches [11], so that the complete segment can be represented as a Norton equivalent

$$
i+G_{N} v=i_{N}
$$

Such equations can be written for the three linear branches, with the currents $i_{\text {left }}, i_{\text {right, }}$, and $i_{C}$. The Norton current $i_{N}$ contains only past information and state variables which have to be updated at each time step. By adding up the three equations (15) a common Norton equivalent of the same form is obtained for the three linear branches.

The solution is based on the secant method, with $i=i_{\text {cor }}$ as unknown and $y=v-v_{\text {cor }}$ as mismatch. Starting with two values of $i$ (taken close to its old value) a new value $i_{\text {new }}$ is obtained, to replace the one with the larger mismatch, from

$$
i_{\text {new }}=\frac{i_{1} y_{2}-i_{2} y_{1}}{y_{2}-y_{1}}
$$

The algorithm has the following steps:

- For $i=i_{1}$ obtain

$$
\begin{aligned}
& i_{\text {ave }}=\frac{i+i^{\text {old }}}{2} \\
& q=q^{\text {old }}+i_{\text {ave }} \Delta t / \Delta l \\
& \dot{q}=i / \Delta l \\
& v_{\text {cor }}=f(q, \dot{q})+\frac{q}{C_{R}} \\
& v \text { from }(15) \\
& y=v-v_{\text {cor }}
\end{aligned}
$$

- Repeat above for $i=i_{2}$.

- Use (16) to obtain $i_{3}$ and iterate to convergence.

- With converged $v$ calculate the reflected wave currents (needed later for $i_{N}$ at other end) and update all state variables.

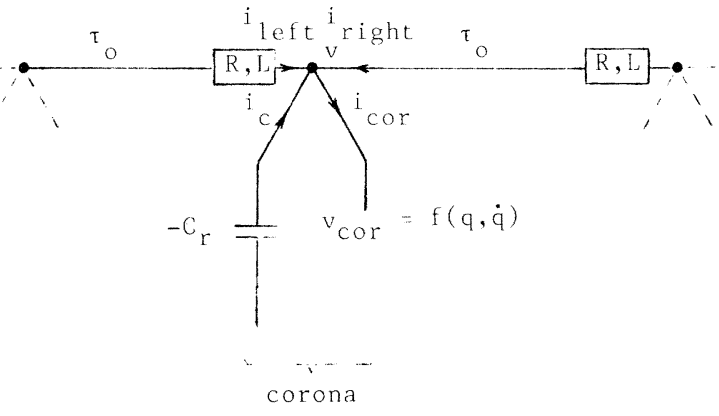

Fig. 13 Travelling wave approach for line modelling.

The above procedure can be generalized for the three phase case when however a multivariable version of the secant method is needed [14].

\section{Simulation of Wave Distortion}

Fig. 14 shows the test case configuration diagram. The lossless line, of length $l$, is divided into small sections $\Delta l(30 \mathrm{~m})$. The capacitance $-C_{r}$ and the corona modelling block are in parallel, at each node of the line sections. The whole transmission line is terminated with the surge admittance $y_{s}=1 / 300 \mathrm{~S}$ at end $B$ and is energized by a Norton equivalent circuit at $A$. The waveshapes in $A$, the midpoint $M$ and at end $B$ are plotted in each test case.

Figures 15 and 16 show the distortion of full wave impulses. Since the surge impedance of the transmission line with corona is smaller than that without corona $(300 \Omega)$, the voltage amplitude in $B$ is slightly higher than in $A$, as expected, and the reflections due to the mismatch of the surge impedance and the terminal resistance can be observed.

Figures 17 and 18 show the distortion of a chopped wave and a wave chopped with oscillations on the tail. Fig. 19 shows the distortion of a sinusoidal wave. In addition to the deformation at the wavefront, the model successfully simulates the opposite polarity corona which appeared before the voltage polarity has changed. The test cases of Figures 18 and 19 show that multilayer corona modelling is necessary.

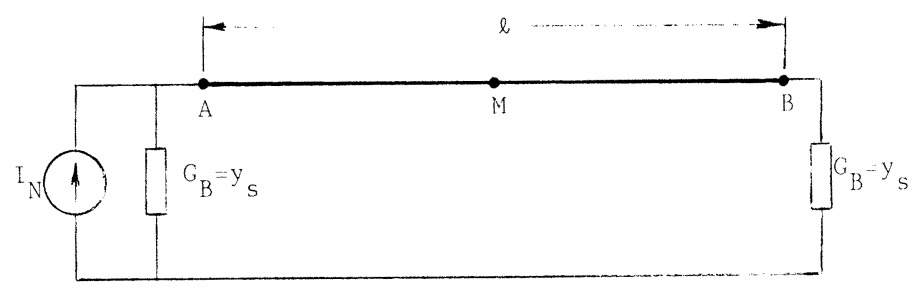

Fig. 14 Test system configuration.

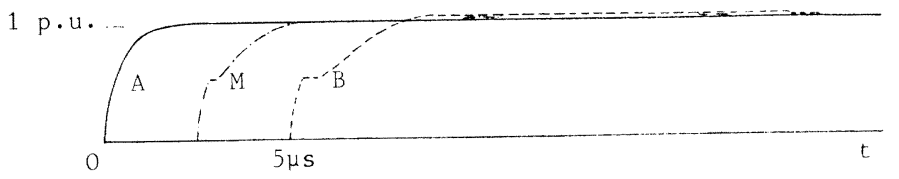

Fig. 15 Distortion of an infinite tail impulse. $A$ - sending end wave $M$ - waveshape at middle of line $B$ - receiving end wave

$(l=1500 \mathrm{~m}$, terminated by surge impedance of $300 \Omega)$ 
1 p.u.

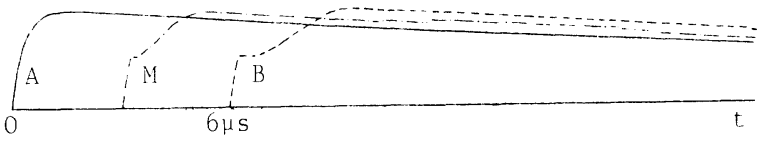

Fig. 16 Distortion of a double exponential impulse on $1800 \mathrm{~m}$ line.

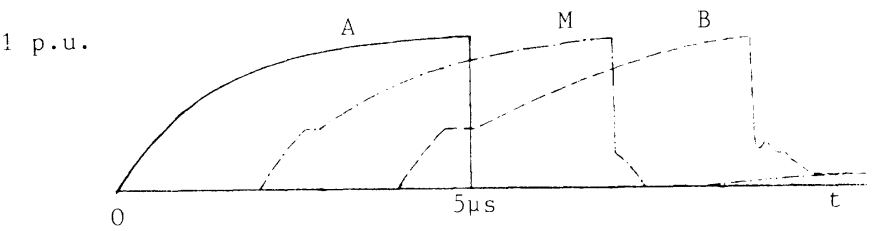

Fig. 17 Distortion of a chopped wave on $1500 \mathrm{~m}$ line.

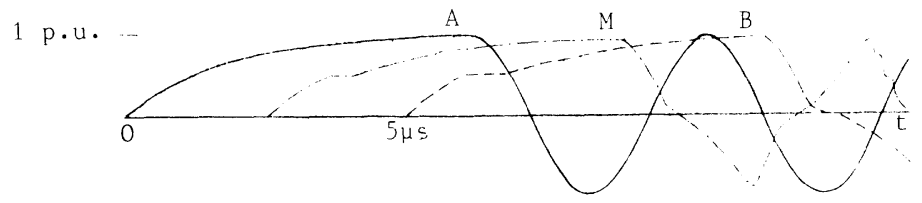

Fig. 18 Distortion of a wave chopped with oscillations at the tail, $l=1500 \mathrm{~m}$.

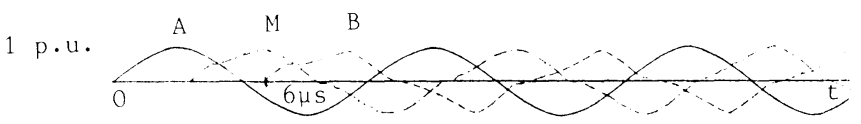

Fig. 19 Distortion of a sinusoidal wave on $1800 \mathrm{~m}$ line.

\section{CONCLUSIONS}

The paper describes a mathematical corona model and transmission line models with corona which can be interfaced with a general purpose Electro-Magnetic Program (EMTP). The model is based on macroscopic field relations which represent a simplified description of complex microscopic phenomena.

The requirement of simplicity has precluded accurate modelling of some complex conditions. In particular, during slow transients, the space charges tend to drift and this creates modelling difficulties, not yet solved in the present study. However, the model has the flexibility to reproduce quite accurately available test results both for the corona phenomenon alone and for the transmission line with impulse waves distorted by corona.

\section{ACKNOWLEDGEMENTS}

Kyong Lee and Joyce Van de Vegte have participated in an early stage of this study. Dr. P. Kundur, of Ontario Hydro, has recognized the significance of this research and given his active support. Dr. A.S. Morched and Dr. D. Shirmohammadi have been helpful in critically reviewing this work. We wish to thank them all for their contributions to this project. Financial support from the Natural Sciences and Engineering Research Council of Canada is gratefully acknowledged.

\section{REFERENCES}

[1] H.W. Dommel, "Digital Computer Solution of Electromagnetic Transients in Single and Multi-Phase Networks", IEEE Trans. on Power Apparatus and Systems, Vol. PAS-88, pp. 388-399, April 1969.
[2] W.S. Meyer and H.W. Dommel, "Numerical Modelling of Frequency-Dependent Transmission Line Parameters in an Electromagnetic Transients Program", IEEE Trans. on Power Apparatus and Systems, Vol. PAS-93, pp. 14011409, Sept J Oct. 1974.

[3] A. Semlyen and A. Dabuleanu, "Fast and Accurate Switching Transient Calculations on Transmission Lines with Ground Return using Recursive Convolutions", IEEE Trans. on Power Apparatus and Systems, Vol. PAS-94, pp. 561-571, March/April 1975.

[4] J.R. Marti, "Accurate Modelling of Frequency-Dependent Transmission Lines in Electromagnetic Transient Simulations", IEEE Trans. on Power Apparatus and Systems, Vol. PAS-101, pp. 147-155, January 1982.

[5] G. Gela, W. Janischewskyj and A. Semlyen, "Modelling of Transmission Lines Including Corona and Frequency Dependence of Parameters", International Electrical and Electronics Conference and Exposition, Toronto, 1975, Conference Digest, pp. 152-153. Details in G. Gela, "Propagation of Surges on Transmission Lines Including Frequency and Voltage Dependence of Parameters", M.A.Sc. Thesis, 1975, Department of Electrical Engineering, University of Toronto.

[6] C. Gary, A. Timotin and D. Cristescu, "Prediction of Surge Propagation Influenced by Corona and Skin Effect", IEE Proceeding, Vol. 130, Pt. A, No. 5, July 1983, pp. 264-272.

[7] K.C. Lee, "Non-Linear Corona Models in an Electromagnetic Transients Program (EMTP)", IEEE Transactions on Power Apparatus and Systems, Vol. PAS-102, pp. 2936-2942, September 1983.

[8] C. Gary and M. Moreau, "L'effet de Couronne en Tension Alternative", Eyrolles, 1976.

[9] R.J. Harrington and M. Afghani, "Implementation of a Computer Model to Include the Effects of Corona in Transient Overvoltage Calculations", IEEE Trans. on Power Apparatus and Systems, Vol. PAS-102, pp. 902-910, April 1983.

[10] P. Sarma Maruvada, H.Menemenlis, and R. Malewski, "Corona Characteristics of Conductor Bundles Under Impulse Voltages", IEEE Trans. on Power Apparatus and Systems, Vol. PAS-96, pp. 102-115, Jan JFeb. 1977.

[11] A. Semlyen and A. Deri, "Time Domain Modelling of Frequency Dependent Three-Phase Transmission Line Impedance", IEEE Trans. on Power Apparatus and Systems, Vol. PAS-104, 1985.

[12] F.A. Liurko, "Characteristics of Corona in Cylindrical Geometry under Various Voltage Shapes", Bulletin of the Polytechnic Institute of Leningrad, No. 1, 1954, pp. 132137.

[13] A. Semlyen, "Electro-Magnetic Transients on Overhead Transmission Lines - An Overview", Canadian Electrical Engineering Journal, Vol. 9, No. 4, 1984, pp. 146-151.

[14] J.M. Ortega and W.C. Rheinboldt, "Iterative Solution of Nonlinear Equations in Several Variables", Academic Press, 1970.

[15] G. Strang, "Linear Algebra and Its Applications", 2nd Ed., Academic Press, 1980. 


\section{APPENDICES}

Appendix 1. Analysis of Breakdown

If the ionized area is assumed to be perfectly conductive then, with $E_{C R}$ at its surface, we have

$$
v=E_{C R} X \ln \frac{R}{X}
$$

where $E_{C R}$ is a function of $X$. A breakdown occurs when $d v / d X=0$ or, from (A-1),

$$
\frac{d E_{C R}}{d X} X \ln \frac{R}{X}+E_{C R}\left(\ln \frac{R}{X}-1\right)=0
$$

If $E_{C R}=$ const then, from (A-2),

$$
\ln \frac{R}{X}-1=0
$$

This yields the well known result:

$$
X_{C R}=\frac{R}{e}
$$

which is too large to be acceptable. For $X_{C R}$ to be smaller, the second term in (A-2) must be positive and, therefore, the first term becomes negative, or

$$
\frac{d E_{C R}}{d X}<0
$$

This shows that $E_{C R}$ must be a decreasing function of $\boldsymbol{X}$.

For a constant $E_{C E}$, wher (A-2') applies, equation (A-1) yieids the breakdown voltage $E_{C H} \frac{R}{e}$. When $E_{C R}$ is a decreasing function of $X$, equation (A-1) yields a smaller value for the breakdown voltage, because both of its main factors are smaller.

\section{Appendix 2. Convergence Analysis of Sweeplng}

The eigenvalue analysis assumes linearity, i.e. the following equations written for Fig. 12:

$$
\begin{aligned}
& L \dot{x}_{2}=v_{A}-x_{3} \\
& C \dot{x}_{3}=x_{2}-x_{4} \\
& L \dot{x}_{4}=x_{3}-x_{5} \\
& \cdot \\
& C \dot{x}_{N-2}=x_{N-3}-x_{N-1} \\
& L \dot{x}_{N-1}=x_{N-2}-v_{B}
\end{aligned}
$$

Trapezoidal discretization and the change of variables

$$
e_{k}=x_{k}-x_{k \infty}
$$

where $e_{k}$ is the error and $x_{k \infty}$ the converged solution, yields:

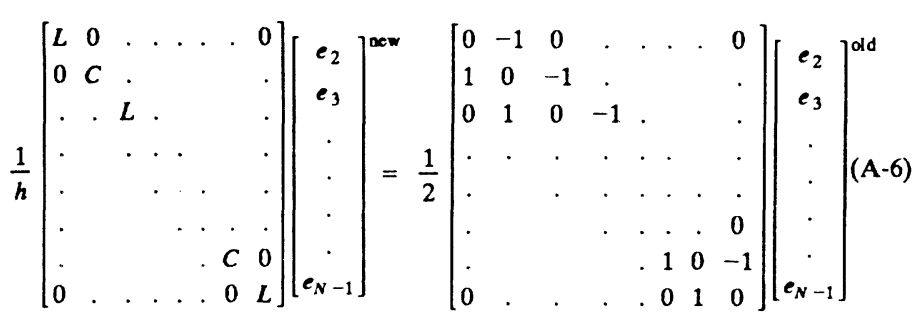

or, in normalized form:

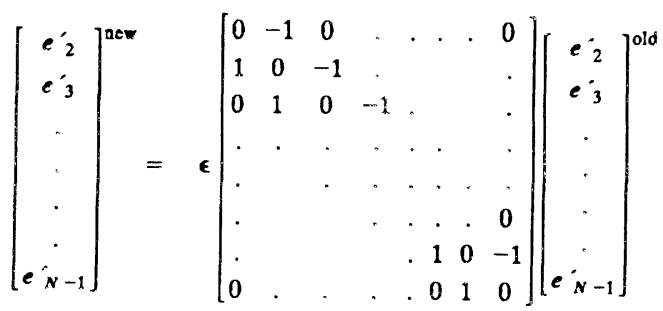

where

$$
\epsilon=\frac{h}{2 \tau_{0}}<1
$$

In $(A-8) \tau_{0}=\sqrt{L C}$ is the travel time for each section.

With matrix notation (A-7) becomes

$$
e^{\text {new }}=M e^{\text {old }}
$$

For iterative solution of (A-9) we write (see [15]):

$$
S e^{\text {new }}=T e^{\text {old }}
$$

where for right sweep (from left) we have:

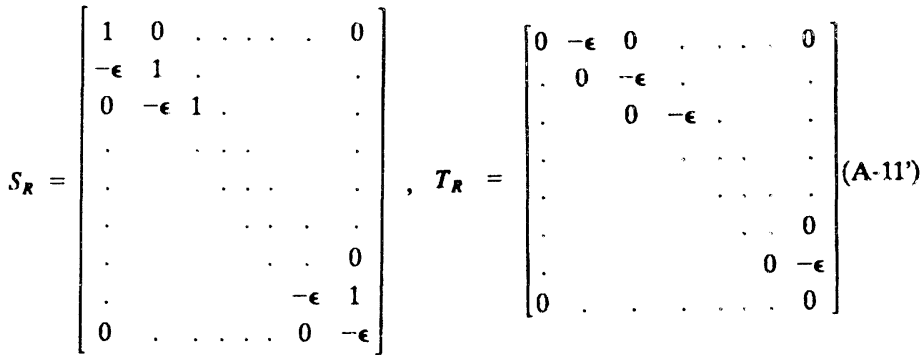

and for left sweep:

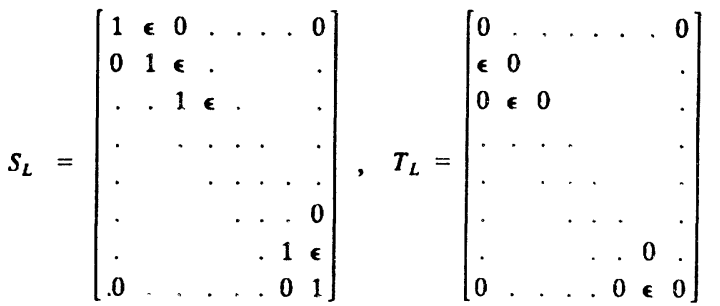

$(A-11 ")$

For convergence analysis we take the eigenvalues of

$$
M_{R}=S_{R}^{-1} T_{R}
$$

and

$$
M_{L}=S_{L}^{-1} T_{L}
$$

Denote

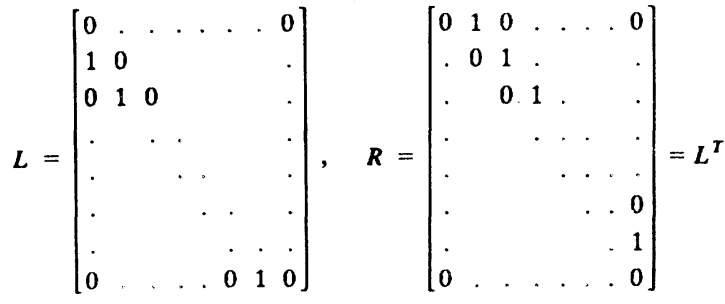

Then, clearly,

$$
M=\epsilon(L-R)
$$




$$
\begin{gathered}
\left\{\begin{array}{l}
T_{R}=-\epsilon R \\
S_{R}=I-\epsilon L
\end{array}\right. \\
\left\{\begin{array}{l}
T_{L}=\epsilon L \\
S_{L}=I+\epsilon R
\end{array}\right.
\end{gathered}
$$

Accordingly, (A-12) yields for $\epsilon \ll<0$ :

$$
\begin{aligned}
& M_{R} \approx(I+\epsilon L)\left(-\epsilon L^{T}\right)=-\epsilon L^{T}-\epsilon^{2} L L^{T} \\
& M_{L} \approx\left(I-\epsilon L^{T}\right)(\epsilon L)=\epsilon L-\epsilon^{2} L^{T} L
\end{aligned}
$$

Substitution of (A-13) into (A-15) yields:

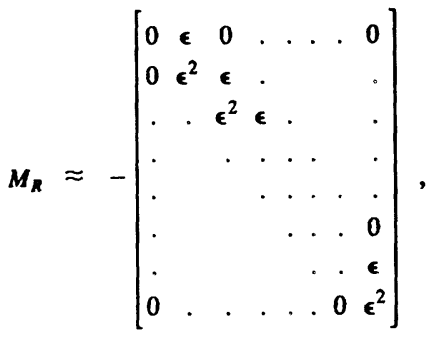

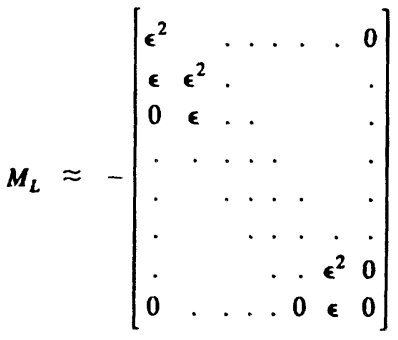

We note that all eigenvalues of both $M_{R}$ and $M_{L}$ are approximately equal to $-\epsilon^{2}$ (except for a single one which is zero). This assures a very good convergence if the time step $h<<\tau_{0}$. The convergence rate is uniformly:

$$
\begin{aligned}
& -\epsilon^{2} \text { for a single sweep } \\
& -\epsilon^{4} \text { for a double or complete (go and return) sweep }
\end{aligned}
$$

For example, if $h=\tau_{o} / 2$, then $\epsilon=0.25$ and the convergence rate is $1 / 16$ for a single sweep and $\approx 0.004$ for a double sweep. If $h=\tau_{0} / 5$, then $\epsilon=0.1$, and the convergence rate is $10^{-2}$ and $10^{-4}$ for a single or double sweep, respectively.

If equations (A-4) were solved independently, say, by parallel processing, i.e. with the inputs being old values of the variables (without the benefit of sweeping which provides an updated input on one side), then the convergence rate would have been only of the order of $\epsilon$ and twice as many calculations would have been need to achieve the same accuracy of convergence.

\section{Discussion}

George Gela, (The Ohio State University): The paper presents a valuable lesson in modeling of corona on overhead lines for the purposes of calculation of propagating transients. It also poses a number of interesting questions. Some of these are explored here in detail.

Foremost is the question of the physical significance of the mathematical equations proposed to account for corona, as well as the possible implications of those equation. The space near the conductor is divided into three regions:

- external to the fictitious cylinder of radius $\mathrm{R}$; not much interest is placed in this region;

- between the cylinder of radius $\mathrm{R}$ and one at radius $\mathrm{X}$; this is termed by the authors the "nonionized region";

- between the cylinder of radius $X$ and the conductor itself (radius r).

As in most studies, the region of active ionization (where ions of polarity identical to that of the conductor are produced as a result of electronmolecule collisions) is neglected. Therefore, the region between $\mathrm{r}$ and $\mathrm{X}$ contains ions already produced in the active ionization layer, and drifting between $\mathrm{r}$ and $\mathrm{X}$ (radially in or out due to the assumption of cylindrical geometry) under the action of the local electric field (also radial). When voltage is applied to the conductor and corona onset is reached, ions initially drift outward since they are of the same polarity as the conductor. With no reversal in field direction, i.e. no voltage dips $(\mathrm{dv} / \mathrm{dt}$ does not change sign), these ions would gradually move away from the conductor, thus pushing the coordinate $\mathrm{X}$ far away, possibly to locations where the authors' assumption of cylindrical geometry is not valid. The authors avoid this difficulty by limiting the applicability of their model to transients which are not too slow, i.e. for which successive reversals in field direction would occur frequently. Yet, Fig. 15 of the paper corresponds precisely to the forbidden case of no field direction reversals at all. The discusser's understanding of Fig. 15 is, therefore, that the model attempts to compute the wavefront only where the voltage is continually changing (rising), but calculations should not be continued too far into the flat top of the wave. Consequently, the conclusion regarding the correctness of the overshoot may appear to correspond to expectations, but it in fact cannot be justified fully.

For cases with input voltage waveforms which do not exhibit oscillation, the following further questions arise. It is clear that the ionized region will continue to expand, i.e. coordinate $\mathrm{X}$ will continue to increase until a field direction reversal. This obviously cannot be permitted to a significant extent, since the assumptions of cylindrical geometry and of negligible importance of ion drift will both soon be violated. On the other hand, the travel time on long lines may be quite large. Consequently, what in the opinion of the authors are the limits on line lenghts imposed by the proposed fast transient model? Would these limitations be less severe for line terminations which result in negative reflection coefficients (i.e. field direction reversals take place at the termination)?

The line length is important also in another respect. For short lines, such as those for which the proposed corona model may be applied safely, the section length must be quite small ( $30 \mathrm{~m}$ in the paper). In his work a decade ago, the discusser found (A) that the commonly employed Carson's model of ground return is inadequate because, among other problems, it neglects displacement currents. Do the authors expect to find it necessary to include in their future work refinements to frequency modelling of the short line section?

A short comment pertains to the authors' expression (Eq. 1) for field $\mathrm{E}$ in the ionized region. While Eq. 1 may be computationally convenient, the discusser would like to point out that the Townsend's closed-form equation with constant ionic mobility

$$
E=\frac{k_{1}}{X} \sqrt{X^{2}+k_{2}}
$$

where $\mathrm{k}_{1}$ and $\mathrm{k}_{2}$ are constants related (B) to geometry and applied voltage, and $\mathrm{X}$ is the radial coordinate, exists and may also yield itself to ready investigation. Also, the ciritical field intensity $\mathrm{E}_{\boldsymbol{O}}$ at the conductor surface depends on the conductor radius $r$ :

$$
\mathrm{E}_{\mathrm{O}}=31\left(1+\frac{0.308}{\sqrt{\mathrm{r}}}\right)
$$

These details are secondary at this time and are listed for future reference

Lastly, the discuser would like to congratulate the authors for their success in devising a corona model which is dynamic in approach Although the model is not free from difficulties, it certainly is a significant improvement over the rigid types available until now.

\section{REFERENCES}

[1] Gela, G., Janischewskyj, W. and Semlyen, A., "Modeling of Transmission Lines Including Corona and Frequency Dependence of Parameters," International Electrical and Electronics Conference and Exposition, Toronto, 1975, Conference Digest, pp. 152-153. 
Details in G. Gela, "Propagation of Surges on Transmission Lines Including Frequency and Voltage Dependence of Parameters," M.A.Sc. Thesis, 1975, Department of Electrical Engineering, University of Toronto.

[2] Janischewskyj, W. and Gela, G., "Computation of Electric Fields Surrounding HVDC Transmission Lines in Corona," Japan- U.S. Science Seminar on Detection and Control of Electric Field and Space Charge in Electrical Environmental Problems, Kyusyu University, Japan, October 1984.

\section{Manuscript received July 23, 1985.}

T. Teranishi and S. Yanabu (Toshiba Corporation, Kawasaki, Japan): The authors should be commended for the contributions in providing a valuable technique for corona modeling for EMTP.

For the insulation coordination of transmission systems, it is important to understand accurately damping of a surg traveling on transmission lines.

We are interested in the resonance phenomena of transformer windings to an oscillating surge from transmission lines. An oscillating surge is caused by, for example, a lightning near a substation and reflection of the surge between the lighting point and a transformer terminal. In this case, the voltage amplitude induced in the transformer winding depends greatly on the damping factor of the oscillating surge striking the transformer terminal.

Corona discharges is considered to be a large factor determining the damping of surges. Therefore we would like to know the damping factor of oscillating surges due to loss of corona.

\section{Manuscript received July, 29, 1985.}

A. T. Johns (University of Bath, Bath, England): The authors have produced interesting computational methods for corona modeling. Some points on which the authors are invited to comment are as follows

a) The need for discretisation will degrade the accuracy of realization of the simulation relative to a distributed line simulation. To what extent is such degradation likely to be significant?

b) From the paper, the effect of corona on the surge waveshapes would appear to be marked only in very long lines of a length considerably in excess of the norm. With this in mind, can the computational complexity and the concomitant increase in digital computing time necessary to implement the methods be justified?

c) Corona discharge is significantly affected by climatic conditions which, over such long line lengths, can vary from one point to another. Has the effect of climate been considered?

d) The corona model would appear to be oversimplistic because, in EHV applications, the use of bundled conductors is widespread. Could the authors comment on the effect of bundled conductors and can they confirm that the situation they propose gives realistic results in these essentially more practical situations?

Manuscript received August 1, 1985.

M. T. Correia de Barros (Instituto Superior Técnico, Lisboa, Portugal): The authors are to be congratulated for their important work. I would greatly appreciate if they could clarify some points about their paper.

1). As the proposed model is "developed from macroscopical physical laws," it would be very interesting to emphasize the relation between the model parameters (A, a, B, b and eventually $\mathrm{k}$ ( on one side, and the practical parameters of corona (polarity, conductor radius, humidity etc.) on the other. The physical meaning of $E_{C R}$ and $E_{c r}$, as function of $x$, would also be helpful to the physical understanding of the model. 2). When the corona model is used for transmission line modeling, the different parameters are chosen to fit experimental $q-v$ curves. Are they kept constant for all branches along the transmision line?

If the answer is yes, then the corona dynamics is not being taken into account.

As a matter of fact, it is well known that, for fast surges, the $q-v$ curve depends on the applied voltage rise rate, which changes along the line as a consequence of corona itself, among other reasons.

3) As the type of wave distortion is directly related to the shape of the charge - voltage diagram $|\mathbf{A}|$, it would be interesting to relate the distorted voltage waveforms presented in this paper to the $\mathrm{q}-\mathrm{v}$ curves considered in each case.

4) The authors mention the problem of parasitic oscillations being excited by high frequency input and discuss the problem of removing them.
In reference $|\mathrm{A}|$ this problem is also discussed and it is shown how those oscillations can be smoothed out by the time integration algorithm itself.

\section{REFERENCE}

[A] M. T. Correia de Barros, J. F. Borges da Silva, "A nonlinear propagation algorithm for transmission lines," 8th Power Systems Computation Conference, Helsinki, 1984.

Manuscript received August 23, 1985.

A. Semlyen (University of Toronto, Toronto, Canada) and Huang WeiGang (Tsinghua University, Beijing, China): We would like to thank the discussers for their interest in our paper, their questions and comments.

At present, simulation of corona phenomena on transmission lines is normally performed using $q-v$ curves which usually represent only a halfcycle external manifestation of the complex, microscopic, physical phenomena around the conductor. Since the modeling of the latter is not practically feasible, the paper presents a macroscopic model which adequately reproduces a given $q-v$ curve. This, however, is only a particular manifestation of the macroscopic model; under conditions of a general transient, the model is ideally still supposed to perform according to reality. Unfortunately, available measurements, whether in the form of $q-v$ curves or attenuation of impulse waves, do not provide enough information for the effect of corona on general transients. On the other hand, it is clear that our present model is still not sufficiently flexible to be accurate enough under all practical conditions. In particular, for the practically of computations, the dynamics of space charges has been very much simplified. Therefore, the effect of corona on slow transients will not be adequately simulated.

$\mathrm{Dr}$. Gela refers in his discussion to the problem of charge dynamics. In the case of the fast impulse of Fig. 15, the total time considered is $25 \mu \mathrm{s}$. With a mobility of $1.5(\mathrm{~cm} / \mathrm{s}) /(\mathrm{V} / \mathrm{cm})$ (see Reference [A]) and a field intensity of less than $30 \mathrm{kV} / \mathrm{cm}$, the ions at the front $\mathrm{X}$ of the ionized region will move, in this time span, by less than $1.125 \mathrm{~cm}$, which is quite insignificant. Thus, the model appears to be adequate for fast transients. However, as pointed out by the discusser, in the case of slow transients a significant drift will occur if there is no polarity reversal. Slow transients, however, are generally oscillatory so that, again, the adequacy of the model is plausible for most practical cases. Nonetheless, it is clearly still important that an improved, dynamic corona model be developed.

Dr. Gela's concern about the adequcy of Carson's model for ground return representation is, in our appreciation, perfectly legitimate for chopped waves where the associated frequency is very high. An impulse of $1 \mu \mathrm{s}$ front time has, however, an associated frequency of only $1 \mathrm{MHz}$, so that Carson's approach (or a related approximation [B]) is still valid. The fact that the discretization into $0.1 \mu \mathrm{s}$ segments corresponds to higher natural frequencies is not significant in this respect, because the induced high frequency oscillations are of parasitic nature and have to be suppressed.

We note that Townsend's equation can be written (with $\eta=E ; \kappa_{1}$ and $\xi=x\left(x_{2}\right)$ in the normalized form

$$
\eta=\frac{\sqrt{1+\xi^{2}}}{\xi}
$$

while equation (3) of the paper, pertaining to the field in the ionized region, can be normalized (with $\eta=E_{c r} / \alpha$ and $\xi=\alpha x / b$ ) to

$$
\eta=1+\frac{1}{\xi}
$$

Equation $(\mathrm{C} 2)$ can be considered as the lowest order asymptotically accurate rational approximation of equation (C1).

In response to Messrs. Teranishi and Yanabu we wish to mention the experience of one of the authors with a $500 \mathrm{kV}$ transmission line in China. The wave formed by backflashover and entering the substation is short (less than $10 \mu \mathrm{s}$ ) and has a very short and high peak; oscillations appear due to multiple reflections. The damping of these surges can be determined by the method of Reference [C] combined with the procedure in this paper.

Our response to the questions of Dr. Johns are the following.

Discretization does not pose any significant problem of accuracy as long as it is sufficiently dense but, as the discusser rightly points out, it is of concern with respect to the cost of computation. Therefore, where corona itself may not be of concern, one should use the traditional traveling wave approach with frequency dependent line modeling, at least for the part of the line not affected by corona. If the meterorological conditions along the line are known, one could possibly take these into ac- 
count by choosing appropriate (local) corona parameters. The problem of corona on bundled conductors is certainly a very complex one. It is clear that the assumption of cylindrical geometry of our paper does not apply in this case. We believe, however, that there exists an equivalent conductor which can be used to computationally replace the bundle. This and the problem of climatic effects will require a significant amount of research for their solution.

To the questions and remarks of Dr. Correia de Barros we offer the following answers.

We have developed simple analyticaal relations between the model parameters $A, B, a, b$ and some points and slopes of the $q-v$ curve. Using Eq. (1) of the paper and an Eq. analogous to (4), we obtain

$$
\mathrm{X}=\left[\frac{q}{2 \pi \epsilon_{o}}-B\right] / A
$$

Then, integrating $E$ from $r$ to $X$ then to $R$, yields

$$
v=\frac{q}{2 \pi \epsilon_{0}} \ln \frac{R}{\mathrm{X}}+a(\mathrm{X}-r)+b \ln \frac{\mathrm{X}}{r}
$$

which has the derivative

$$
v^{\prime}=\frac{d v}{d q}=\frac{1}{2 \pi \epsilon_{0}}\left[\ln \frac{R}{\mathrm{X}}-\frac{q}{2 \pi \epsilon_{\mathrm{O}} \mathrm{X} A}+\frac{a}{A}+\frac{b}{\mathrm{XA}}\right]
$$

The parameter identification from these equations is not immediate and requires a carefully devised computer program.

The expressions of $E_{\mathrm{CR}}$ and $E_{c r}$ in terms of $\mathrm{X}$ and $x$, respectively, are approximations chosen for computational convenience (see the response to Dr. Gela): we also know that $E_{\mathrm{CR}}$ must decrease with $x$ to satisfy some breakdown test data. We found it very revealing how in the discusser's work (Ref. D) the attenuation of the frontal slope of an impulse is shown to be directly related to the curvature of the $q-v$ curve. This relation is very important because it shows that both $q-v$ curves and impulse test result can potentially be used for the parameter identification in a macroscopic physical corona model.

Dr. Correia de Barros questions whether different parameters have to be used in the corona models along the line to correspond to the varying speed of the transient: in our approach, corona dynamics, as it affects the $q-v$ curve, is taken into account by $E_{\mathrm{CR}}$ being a function of $q$ the time derivative of $q$. For such a model, the $q-v$ curves will result different along the line, but they are not explicitly represented in the line modeling equations.

The trapezoidal rule is known to produce numerical oscillations if the time step is not small enough. We consider that the better integration techniques presented in reference [A] of the discussion are of special interest because they solve this problem. We wonder, however, whether oscillations are not excited also by the physical descretization involved in the line representation?

\section{REFERENCES}

[A] P. S. Maruvada and W. Janischewskyj, "Analysis of Corona Losses on DC Transmission Lines: I - Unipolar Lines," IEEE Trans. Power App. and Syst. Vol. PAS-88, pp. 718-731, May 1969.

[B] A. Deri, G. Tevan, A. Semlyen and A. Castanheira, "The Complex Ground Return Plane, a Simplified Model for Homogeneous and Multi-Layer Earth Return," IEEE Trans. Power App. and Syst. Vol. PAS-100, no. 8, pp. 3686-93, August 1981.

[C] J. Osawa, E. Ohsaki, M. Ishii, S. Kojima, H. Ishihara, T. Kouno, T. Kawamura, "Lightning Surge Analysis in a Multi-Conductor System for Substation Insulation Design," IEEE Trans. Power App. and Syst. Vol. PAS-104, no. 8, pp. 2244-2251, August 1985.

[D] M. T. Correia de Barros and J. F. Borges de Silva, "Computer Simulation of the A. C. Corona Effect Above the Critical Gradient," Proceedings of the Seventh Power Systems Computation Conference, Lausanne, July 1981, pp. 894-899.

Manuscript received October 1, 1985. 